\title{
INDOOR SPACE LOCATION MODEL BASED ON LOCATION SERVICE
}

\author{
Yan Zhou $^{\text {ab }}$, Guixiang Zeng ${ }^{\mathrm{a}}{ }^{*}$, Yueying Huang ${ }^{\mathrm{a}}$, Xiaoxia Yang ${ }^{\mathrm{c}}$ \\ ${ }^{a}$ School of Resources and Environment, University of Electric Science and Technology of China, 2006 Xiyuan Avenue, West Hi- \\ tech Zone, Chengdu, Sichuan 611731, China; (zhouyan_gis@uestc.edu.cn, zengguixiang11@sina.com, 1229293139@qq.com ) \\ ${ }^{\mathrm{b}}$ Institute of Remote Sensing Big Data, Big Data Research Center of University of Electric Science and Technology of China \\ ${ }^{c}$ Colloge of Earth Sciences, Chengdu University of Technology, Chengdu, 610059, China
}

\section{Commission VI, WG VI/4}

KEY WORDS: IndoorGML, Indoor LBS, Geometry, Topology, Semantics, Indoor Space Location Model

\begin{abstract}
:
Location is the basis for the realization of location services, the integrity of the location information and its way of representation in indoor space model directly restricts the quality of location services. The construction of the existing indoor space model is mostly for specific applications and lack of uniform representation of location information. Several geospatial standards have been developed to meet the requirement of the indoor spatial information system, among which CityGML LOD4 and IndoorGML are the most relevant ones for indoor spatial information. However, from the perspective of Location Based Service (LBS), the CityGML LOD4 is more inclined to visualize the indoor space. Although IndoorGML is mainly used for indoor space navigation and has description (such as geometry, topology, and semantics) benefiting for indoor LBS, this standard model lack explicit representation of indoor location information. In this paper, from the perspective of Location Based Service (LBS), based on the IndoorGML standard, an indoor space location model (ISLM) conforming to human cognition is proposed through integration of the geometric and topological and semantic features of the indoor spatial entity. This model has the explicit description of location information which the standard indoor space model of IndoorGML and CityGML LOD4 does not have, which can lay the theoretical foundation for indoor location service such as indoor navigation, indoor routing and location query.
\end{abstract}

\section{INTRODUCTION}

In recent years, with the development and popularization of wireless communication, perceived positioning and internet technology, location-based services have a lot of applications in traffic, logistics, emergency response and people's daily life. The hotspot of the research in the field of international location service has also been derived from the outside to the indoor space, and the indoor space model is the prerequisite and the key to realize the location service. Location play an important role in the field of location awareness and context-aware systems. Especially in the ubiquitous field of computing, where location is often considered an important source of context (Schilit, 1995). The objects of the outdoor environment are usually located in a unified Euclidean coordinate system, so that it can be absolutely positioned, however, indoor positioning is influenced by the indoor environment, the location of indoor spatial entity only through the local coordinates to realize relative positioning. In the process of finding the road or inquire some place in indoor space, influenced by human cognition, the way of describing the absolute direction of the "east, south, west, north" is no longer suitable for the representation of the indoor space, and the approach of "front, back, left, right" is more suitable for the relative positioning which conform with the human cognition for indoor space. At present, many scholars have studied the indoor space model in the world. The existing indoor space model only represents part of indoor spatial information, such as geometric model, symbolic model and semantic model, the scope of application of such model is limited. IndoorGML is a standard data model to represent, store and exchange indoor spatial information, which contains the geometric, topological and a small amount of semantic information of the indoor spatial unit, but lacks the description of location-related semantic location information such as "upstairs", "downstairs" and "opposite". In this paper, explicit representation of indoor location information consisting of indoor geometric location and semantic location is presented based on the IndoorGML standard, and the model proposed in this paper integrates the semantic relative location information such as semantic direction and semantic distance to construct an indoor space model suitable for positioning, navigation and route planning.

The rest of the paper is organized as follows: section 2 reviews recent research progress on modeling locations. In section 3 , we explain the basic concepts of IndoorGML. And in section 4, we propose our model based on the IndoorGML. A simple use case of proposed model is shown in section 5. Finally in section 6, we conclude this paper and put forward the work to be done in the future.

\section{RELATED WORK}

Indoor space model, as well as spatial knowledge representation, has attracted much attention in indoor LBS community. Earlier indoor space models attempt to differentiate the concept of "geometric" and "symbolic" models (Leonhardt, 1998). The object of the symbolic model has a unique name for identifying them in all entities, such as room numbers. On the other hand, locations and objects in geometric models are represented as points, areas or volumes within a reference coordinate system. From the perspective of indoor LBS, according to the structural characteristics of the model, indoor space model can be categorized as geometric space model, symbolic space model and semantic space model.

* Corresponding author 
The geometric space model is usually a geometric description of the indoor entity, which provides accurate geometric information for the model in the form of coordinates. Distance between two objects can be calculated based on their coordinates. In a representative geometric model, the boundary information of the entity is extracted on the basis of IFC, and it is networked to the two-dimensional plane to realize the indoor path planning (LIN et al., 2013). Also, a triangular grid partition of indoor space is proposed (Demyen and Buro, 2007), and an efficient barrier path query is realized by constructing a graph model based on triangular grid. The symbolic space model represents all the objects in the indoor space as symbolic elements with a specific ID tag, generally used to describe the topological relationship between objects. Symbols can be divided into sets and subsets, which can be organized in a hierarchical structure through a small amount of semantic information of indoor space objects (Dürr and Rothermel, 2003). Semantic space model represents the variety of types of entities in indoor space, as well as their properties and relationships, usually associated with the ontology. The indoor navigation ontology model is proposed based on ontology theory (Tsetsos et al., 2006).

The geometric, topological and semantic information of the indoor space model are indispensable to the application of indoor location service. How to comprehensively consider these three types of information is the focus of the research on the indoor space model based indoor location service. The existing standards representing indoor spatial information (such as indoorGML, CityGML LOD4) all contain the geometric, topological, and semantic information of the indoor space, but the CityGML LOD4 is more inclined to visualize the indoor space. Although indoorGML is mainly used for indoor space navigation, however, this standard contains a small amount of semantic information, and lack of explicit representation of location information. Therefore, based on the IndoorGML standard, this paper constructs an indoor space location model considering topological relations, geometric features, and semantic location information.

\section{INDOORGML}

IndoorGML is a standard data model to represent, store and exchange indoor spatial information and a XML application schema (OGC IndoorGML, 2014, Lee et al., 2010) based on GML 3.2.1 (Open Geospatial Consortium, 2007). Unlike CityGML and IFC, IndoorGML focuses primarily on the representation of indoor spatial units (cell) rather than the indoor spatial features such as roof, ceiling, floor, and wall. In IndoorGML, a cell is the smallest organizational or structural unit of indoor space, which can be represented as a room, or a corridor. Entire indoor space is considered as a set of cells and each cell can't overlap with other cells. It is worth noting that each cell has a unique identifier, such as a room number, which is the only implicit semantic symbolic location information in the indoorGML standard model. Therefore IndoorGML provides a standard framework to represent geometry, network, and semantics of cells in indoor space. We briefly explain how to represent these aspects in IndoorGML in this section.

\subsection{Geometric Representation}

Although, geometric representation of cell is not the focus in IndoorGML, there are three options to represent geometry features of indoor space. As shown in Figure 1, the first option is to reference an object defined in other data set such as CityGML, which contains its geometric property. The second option is to include geometric property of cell within IndoorGML data, which is either a solid in $3 \mathrm{D}$ or a surface in $2 \mathrm{D}$. The third option is not to include any geometry property of cell (OGC IndoorGML, 2014).

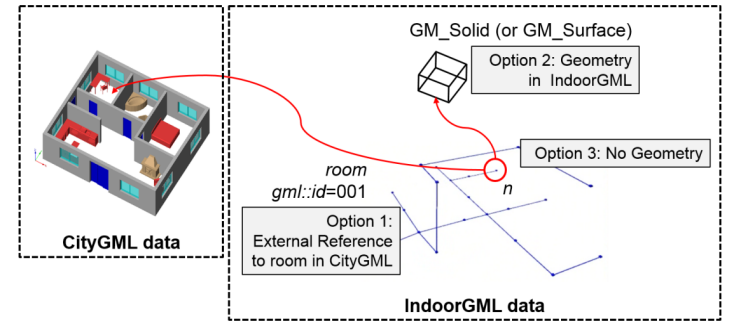

Figure 1. Three options to represent geometry in IndoorGML

\subsection{Network Representation}

Topology is an important part of cell space. Therefore, IndoorGML focuses on the explicit representation of topological relations. The node relationship graph (NRG) (Lee, 2004) represents the topological relationship between indoor spatial unit, such as adjacency and connection. NRG allows abstract, simplify and represent the topological relationships of $3 \mathrm{D}$ spaces in the indoor environment, such as rooms within a building. It can effectively achieve the indoor navigation and routing systems in the complex computing problems. The Poincaré duality (Munkres, 1984) provides a theoretical background for mapping the indoor space to the NRG representing the topological relationship. Figure 2 shows the topographic space and the corresponding NRG (OGC IndoorGML, 2014).

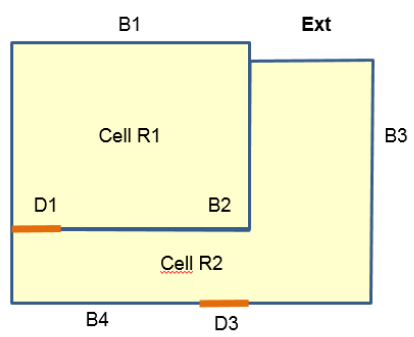

Topographic Space

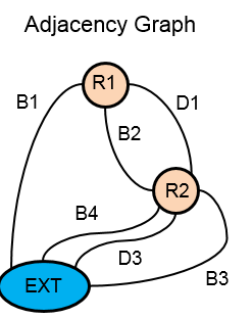

Dual Space
Figure 2. The topographic space and the corresponding NRG

\subsection{Semantic Representation}

Only a small amount of semantic information in IndoorGML can be used to represent symbolic location information for a cell, such as a room number. These semantic information also play a role in classifying indoor spatial units. For example, in the navigation model of IndoorGML, indoor spaces are divided into navigation spaces (such as rooms, corridors, doors) and non-navigational spaces (walls, obstacles, etc.). We can infer the connectivity between the cells based on these small amounts of semantic information. However, the indoor location based service requires more semantic location descriptions, which we describe in the next section.

\section{ISLM}

IndoorGML is a standard data model to represent the geometric characteristics, topological features and semantic features of the indoor space, which laid the model foundation for the indoor navigation, path planning and other location services. However, the standard lacks explicit representation of indoor location information, it is difficult to meet the growing demand for 
indoor LBS. Therefore, in this paper, an indoor space model (ISLM) containing location information is proposed based on IndoorGML. Figure 3 is a UML diagram of the ISLM presented in this paper. As shown in Figure 3, the orange part of the figure is the representation of the indoor space object in the IndoorGML core model, the lower green part is the location information added to the indoor space unit based on IndoorGML. In the ISLM, the location of the spatial cell is defined from both geometric location and semantic location. The detailed definition is described below.

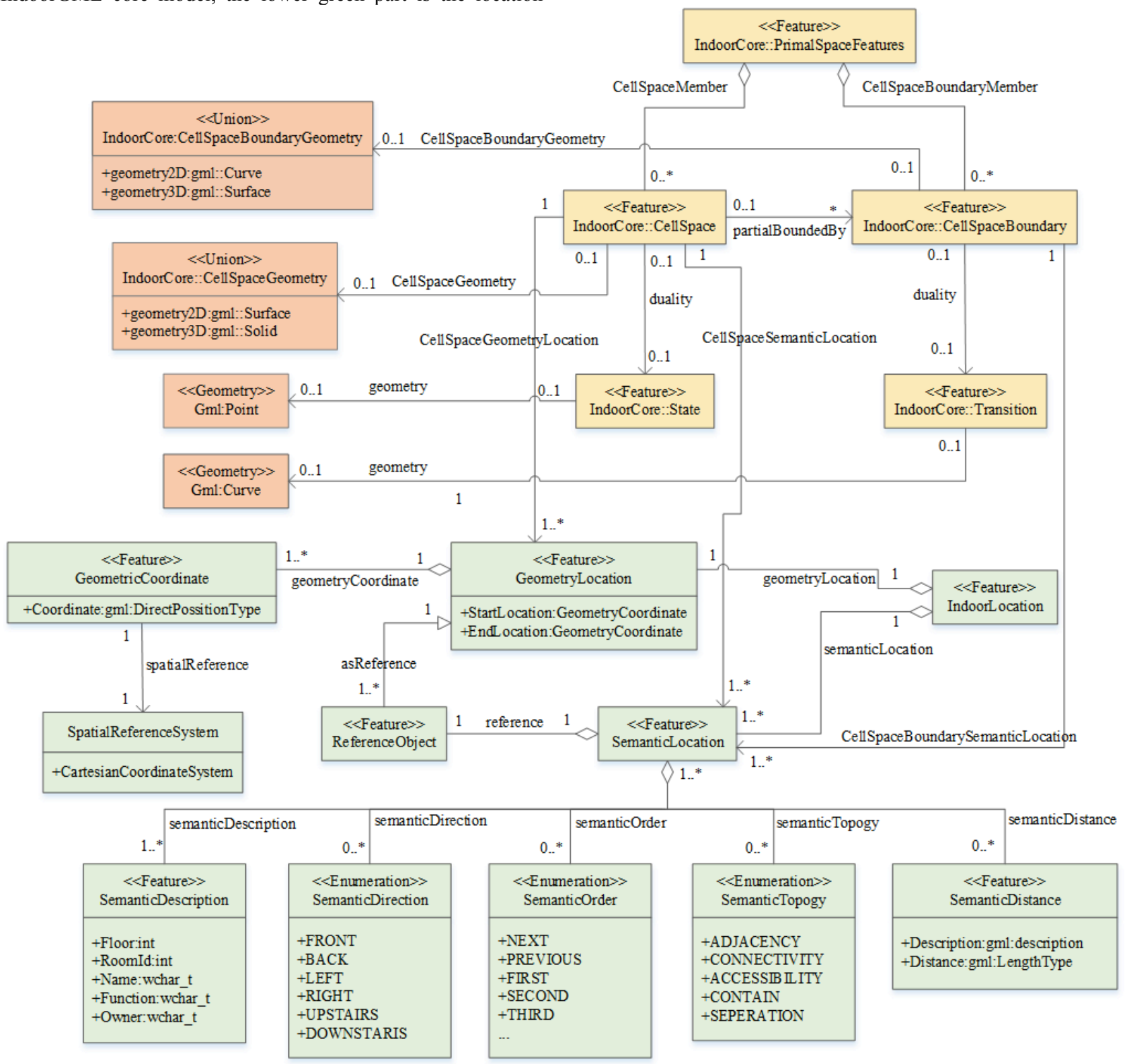

Figure 3. UML diagram of ISLM

\subsection{Topological Representation in ISLM}

As shown in Figure3, the topological relationship between spatial units of ISLM is similar with IndoorGML. According to the notions of geographic features defined by ISO19109, objects in real world can be represented as PrimalSpaceFeatures consisting of CellSpace and CellSpaceBoundary classes. A CellSpace represents one spatial object, such as a room or a corridor. And a CellSpaceBoundary is used to describe the boundary of the each spatial object, usually represent as a door or a wall. The State has association with the corresponding CellSpace class which represents a spatial unit in primal space, which represents a node in dual space depicted in Figure 2. Transition is an edge that represents the adjacency or connectivity relationships among nodes representing spatial objects in primal space, it can be associated with a boundary of a CellSpace in the primary space. The State and Transition in dual space together represent the topological relationships among cells in primary space. Such explicit representation of topological relationship play a key role in the indoor path planning and other location services.

\subsection{Geometric Representation in ISLM and Geometric Location}

The second option defined by IndoorGML (Figure 1) is used to represent geometric information in ISLM, which include geometric property of cell within ISLM, and a cell is either a solid in $3 \mathrm{D}$ or a surface in 2D. When the CellSpace is represented as a gml:Solid in three dimensional space, 
corresponding CellSpaceBoundary is represented as gml:Surface, and when the CellSpace is represented as a gml:Surface in 2 dimensional space, corresponding CellSpaceBoundary is represented as gml:Curve. Also, the geometric information is modelled in CellSpace in ISLM. In dual space, The State is geometric represented as gml:Point and Transition is represented as gml:Curve.

In ISLM, the GeometryLocation allows to define a cell in geographic space that is measured with respect to a standard coordinate system, which is decided by StartLocation and EndLocation represented by geometric coordinate information, such as $\left(\mathrm{X}_{1}, \mathrm{Y}_{1}, \mathrm{Z}_{1}\right)$ and $\left(\mathrm{X}_{2}, \mathrm{Y}_{2}, \mathrm{Z}_{2}\right)$ coordinate. A CellSpace with a geometric location can be used as a reference object for the semantic location of another CellSpace. The geometric information is associated with CellSpace or State instead of CellSpaceBoundary and Transition, since the location of a place that people want to reach usually can be represented as a cell instead of the boundary of a cell, therefore, the geometric information of CellSpce is our focus rather than the location of CellSpaceBoundary. Also, Transition does not contain any geometric location information, it represents the topological relationship between the cells in cellular space, so the hidden relative location relationship can be inferred from the NGR represented in dual space. In this paper, we focus on the explicit relative location relationship of the semantic description, which described detail in 4.3 section.

\subsection{Semantic Location Representation in ISLM}

As shown in Figure3, the IndoorLocation is the root of indoor location information. Based on IndoorLocation, there are two general ways to define indoor location in detail: GeometryLocation and SemanticLocation. The GeometryLocation class is described in Section 4.2, and this section the SemanticLocation class will be introduced in detail.

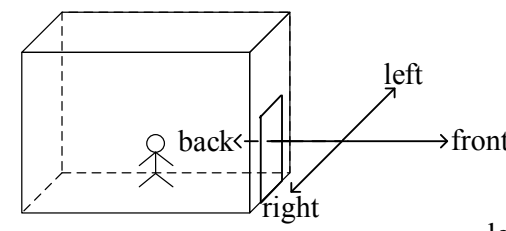

inside

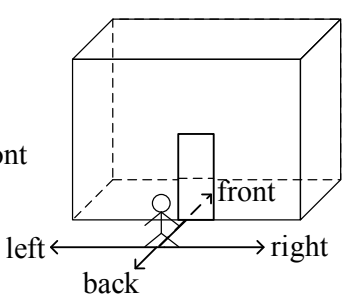

outside
Figure 4. Definition of semantic direction

In the ISLM, SemanticLocation class consisting of SemanticDescription, SemanticDirection, SemanticOrder, SemanticTopology and SemanticDistance represent the relative location of CellSpace and the boundary of CellSpace (CellSpaceBoundary) in primary space. A cell in indoor space (such as room) can be semantically identify by floor number, room number, room name and room type through semantic description information of SemanticDescription class. Users of mobile terminals are usually navigated through the direction of "east, south, west and north" provided by navigation systems in outdoor space, but this approach does not apply to indoor navigation. In the indoor environment, there is more direction to rotate in comparison with the outdoor environment. People in the indoor space is easy to get lost, it is more difficult to identify the direction after the rotation of direction. So from the perspective of indoor spatial cognition, the way describing indirection using "front, back, left, right" is more suitable for the relative positioning in the indoor space, which is depicted in SemanticDirection class. The definition of semantic direction is shown in Figure 4. The direction of the front and back is decided by the user's position relative to the room (inside or outside). In addition, since the spatial entities such as doors and windows are usually represented as the boundary of the cell, it is difficult to use the geometric coordinates for absolute positioning. People can move in indoor space through the first exit, the next exit, the second exit, the last exit and other location information representation for indoor navigation, such way of the description of location is provided by SemanticOrder class. The SemanticTopology class which explicitly defines adjacency, connectivity, accessibility and separation relationships among the indoor cells. In order to achieve the indoor optimal route query, the nearest query and other indoor location service, the semantic distance description (SemanticDistance) is added to the ISLM in this paper. SemanticDisance class defines distance description (including a numerical value of distance) and a semantic description such as:" Three meters away from the known location".

\section{USE CASE OF ISLM}

In this section, a simple example is used to instantiate the description of location information in the ISLM. As shown in Figure 5, a three-dimensional model of laboratory area in the second floor in a building is presented. A room is represented as a cell and a door is represented as the boundary of the room. In this case, the geometric location of the cell is determined by the start position and the end position, which is represented as (200, $50,60)$ and $(100,80,90)$ in the Cartesian coordinate system.

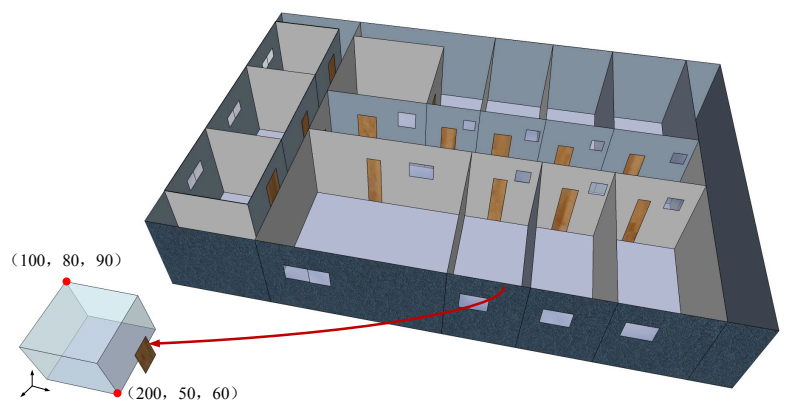

Figure 5. Three - dimensional model of laboratory area

In order to better describe the semantic location information of the spatial unit, the three-dimensional model shown in Figure 5 is mapped to the two-dimensional plane, as shown in Figure 6. The orange part of the figure represents a cell in indoor space, which is defined by SemanticDescription as "A room is located on the 2nd floor and is called the geospatial laboratory, which has the function of accommodating other spatial entities". The semantic location of the green part of the figure can be defined relative to the known location. For example, from room 2014 to the lift, the semantics can be described as follows: Turn left until the corner and then turn right, the location of the last door is the destination. Also, the relative semantic location of room 2105 can be described as "Go out and turn right, the second room on the left" or "a room adjacent to the right side of the room 2014, opposite the room is the target". 


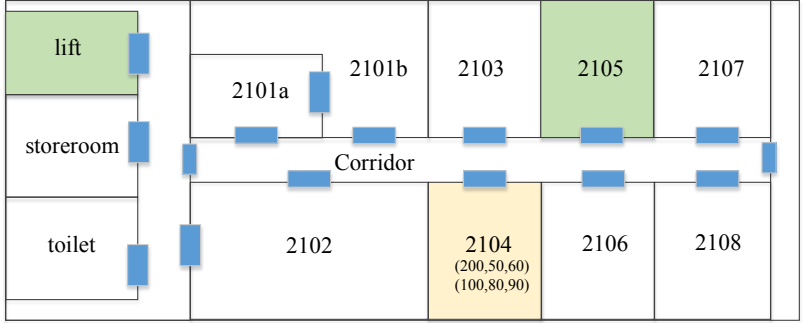

Figure 6. Floor plan of laboratory area

\section{CONCLUSION AND FUTURE WORK}

IndoorGML is an international standard to exchange, store indoor spatial information. It not only contains the geometric and topological information of the indoor spatial unit, but also contains a small amount of semantic information. However, the model lacks the representation of location-related semantic information. Based on the IndoorGML standard, this paper constructs an indoor space location model containing geometric, topological and semantic location information. The model can set the theoretical basis for the indoor space model of indoor location service. Then we need to study the method of data organization of the model on the basis of the ISLM to apply to the specific application, in which the definition of the relevant location information should be further refined or improved to suit the specific location service scenario, such as the definition of semantic distance.

\section{ACKNOWLEDGEMENTS}

This research is supported by National Natural Science Foundation of China (No. 41471332 , 41101354 and 41571392), the National High Technology Research and Development Program of China (No. 2016YFB0502300) and the Fundamental Research Funds for the Central Universities (No. ZYGX2015J113).

\section{REFERENCES}

Schilit WN (1995) A system architecture for context-aware mobile computing. PhD thesis, Columbia University.

U. Leonhardt. Supporting location-awareness in open distributed systems. PhD thesis, Department of Computing, Imperial College London, 1998.

LIN Ya-hong, LIU Yu-shen, Gao Ge,et al. The IFC based Path Planning for 3D Indoor Spaces[J].Advanced Engineering Informatics,2013,27(2):189-205.

Demyen D, Buro M. Efficient Triangulation-Based Pathfinding.[J]. Aaai, 2007, 1338(9):161-163.

Dürr F, Rothermel K. On a Location Model for Fine-Grained Geocast[J]. Lecture Notes in Computer Science, 2003, 2864:1835 .

Tsetsos V, Anagnostopoulos C, Kikiras P, et al. Semantically enriched navigation for indoor environments.[J]. International Journal of Web \& Grid Services, 2006, 2(4):453-478.

Open Geospatial Consortium, OGC IndoorGML, OGC 14005R3, 2014.
Lee, J., Li, K. J., Zlatanova, S., Kolbe, T. H., Nagel,C.,Becker, T., Requirements and Space-Event Modeling for Indoor Navigation, OGC 10-191r1. 2010.

Open Geospatial Consortium, OpenGIS Geography Markup Language (GML) Encoding Standard Version 3.2.1, OGC 07036 (2007).

Lee, J. 2004. A Spatial Access Oriented Implementation of a Topological Data Model for 3D Urban Entities, GeoInformatica 8(3), 235-262.

Munkres, J. R., Elements of Algebraic Topology, AddisonWesley, Menlo Park,CA, 1984. 